\title{
A Versatile System for Computer Integrated Mini-invasive Robotic Surgery
}

\author{
Louai Adhami and Ève Coste-Manière \\ ChIR Medical Robotics group, INRIA Sophia Antipolis (www.inria.fr/chir)
}

\begin{abstract}
This paper presents a versatile system that aims at enhancing minimally invasive robotic surgery through patient dependent optimized planning, realistic simulation and safe supervision. The underlying architecture of the proposed approach is presented, then each component is detailed and illustrated with experimental examples. In particular, an instantiation with the Da Vinci ${ }^{\mathrm{TM}}$ [1] robot for performing cardiac surgery is used as a federating case study.
\end{abstract}

\section{Introduction}

Sophisticated robotic simulators and planners are now found in almost any industrial plant. In parallel versatile medical simulators, image manipulation and augmented reality systems exist for research or even in clinical use. However, the combination of medical imaging and robotics in a single system is rarely encountered. In particular, clinically available robotic CIS systems are mainly associated with surgical robots such as ORTHODOC ${ }^{\circledR}$ for ROBODOC $^{\circledR} 1$ or Carabeamer [2] for Cyberknife ${ }^{\circledR}$, as well as many neurosurgical systems. The main focus of these systems is on planning and execution through proper registration. Optimized port placement is addressed in [3] where the authors plan a minimally invasive CABG with the Zeus ${ }^{\circledR}$ surgical system; however, they do not integrate the models of the robot in the planner and rely on empirical results from [4] for the port placement. Simulation systems that handle robotics in a clinical background are still under development, or are being built upon existing simulation architecture such as [5]. Results in augmented reality are found in a number of research groups (e.g. [6]). Finally, safety issues have always been a central preoccupation in medical robotics.

The need for an integrated system that can combine medical imaging and robotics in a single versatile system is a recent demand that is rising with the advancements of surgical techniques toward increased mini-invasiveness, and with the introduction of robotic tools in the operating room. As stated by [7]: “ . . CIS systems will produce the same impact on surgery that has been realized in computer aided manufacturing", this revolution will go through surgical robotics and will need systems that can efficiently combine the different sources of available information. These needs have formed the major motivation for the work

\footnotetext{
${ }^{1}$ http://www.robodoc.com/eng/robodoc.html
} 
presented in this paper : a computer integrated system for robotic mini-invasive surgery (RMIS). The proposed approach addresses these requirements and can be summarized as follows:

1. Perception: Gather information about the patient, the robot and the environment.

2. Port placement: Determine the best incision sites based on intervention requirements, patient anatomy and endoscopic tools specifications.

3. Robot positioning: Determine the best relative position between the robot, the patient and the operating theater.

4. Verification: Verify the operating protocol and the corresponding robot logic flow.

5. Simulation: Rehearse the intervention using patient and robot data in simulated operating conditions

6. Transfer: Transfer the results to the operating room through proper registration and positioning.

7. Execution: Execute the planned results with visual and computational assistance.

8. Monitoring: Monitor the progress of the intervention and predict possible complications.

9. Analysis: Store the settings and history of the intervention for archiving and subsequent analysis.

All the components of this architecture are integrated into a modular single interfaced system. This paper gives a brief overview of each of the aforementioned steps.

\section{Perception}

A preliminary step to any form of processing is to acquire relevant and usable data. In the case of computer integrated robotic surgery, three sources of information have to be combined. Namely, data about the patient that describes the overall anatomy and gives clear pathologic indications, a model of the robot that can be used to accurately predict its behavior and interaction with its surrounding, and finally a model of the surgical setup or the operating theater that serves to consolidate preoperative planning and simulation with intraoperative execution.

\subsection{Patient Model}

Patient data is mainly obtained from preoperative radiological imaging. An interactive $3 \mathrm{~d}$ segmentation system is used to delineate semantically significant entities for further analysis; e.g., ribs must be isolated and numbered for the planning phase described in section 2 Moreover, $3 \mathrm{~d}$ reconstruction is used to 

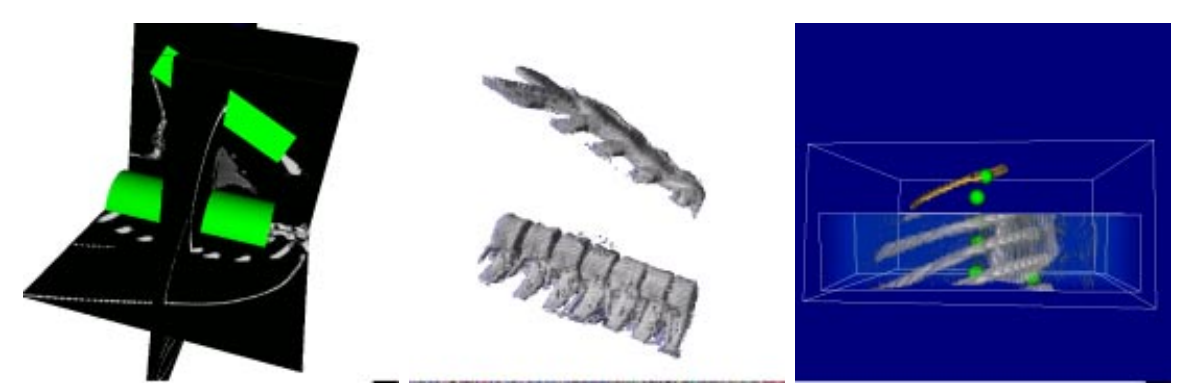

Fig. 1. Interactive 3d segmentation is a successful compromise between versatility and efficiency: (from left to right) 3d manual segmentation of the sternum and backbone, the resulting reconstructed image and an interactive rib growing operation on a volume rendered image.

transform volumetric data into surface data that are easier to visualize and manipulate. Examples of manual and automatic segmentation are shown in figure 1. Under-sampling and triangulation of the segmented data are necessary to enable advanced manipulations such as deformations or active constraints (see section 5].

\subsection{Robot Model}

The kinematics and shape of the robot are inputted using a simple modeling syntax. Standard DH coordinates are used for kinematic description, whereas the physical envelope of the robot is approximated with basic geometric primitives, namely, regular parallelepipeds, cylinders and spheres. This format accepts any general open-chain multi-armed robotic systems; close chains can also be used but are more difficult to model (see figure 2 for examples). The main originality of the format is its ability to model special surgical robotic features such as an endoscopic arm and a variety of surgical tools. A camera can be added anywhere on the robotic chain, and can take different attributes such as a projection matrix. Likewise, different types of tools can be added to model interactions such as cutting tissue.

\subsection{Environment Model}

The environment is represented using a geometric model of the operating theater and approximations of the surrounding material. An extra realism can be added through texture mapping views of the operating room, as shown in figure 2 .

\section{Port Placement}

In robotic MIS, a standard endoscopic configuration composed of a left hand, a right hand and an endoscope is used. An optimization algorithm is used to determine the best entry points from a set of previously segmented admissible ports, 

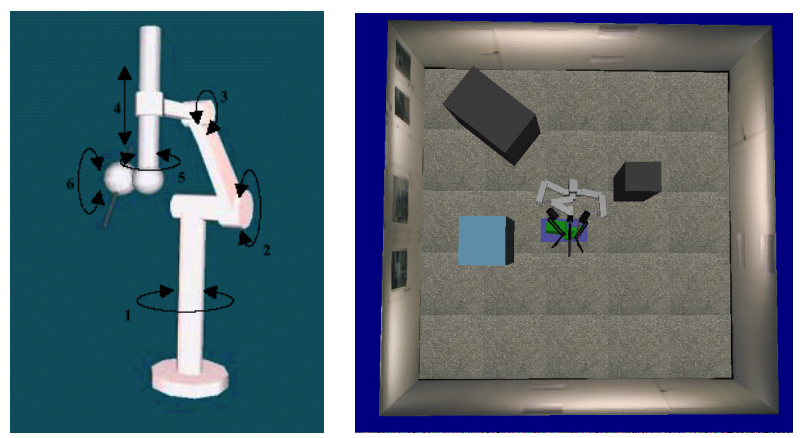

Fig. 2. (left) A prototype of a robot for neurosurgery, (right) Example of an operating room: The surrounding obstacles are important for planning collision free robot movements.
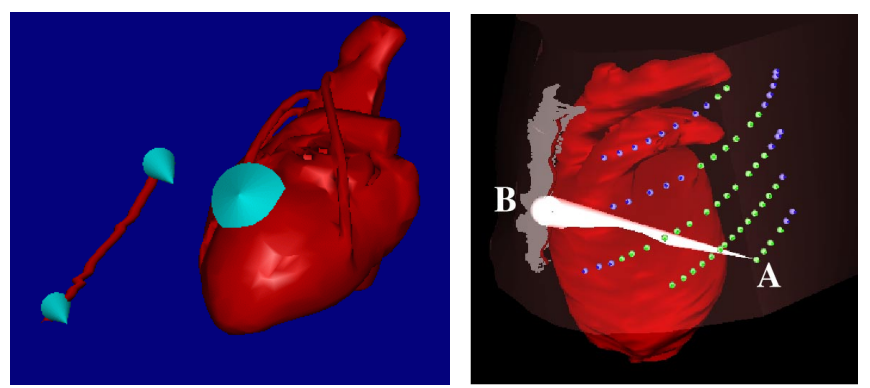

Fig. 3. Optimal port placement: (left) Main syntactic elements (right) Example of a reachability test.

such as the intercostal vectors depicted in figure 3(b). The algorithm is based on a semantic description of the intervention, where the main syntactic elements are target points inserted by the surgeon on the anatomical model of the patient to represent the position, orientation and amplitude of the surgical sites, as shown in figure 3 (a). The semantics of the intervention are translated into mathematical criteria that are subsequently used in an exhaustive optimization to yield the most advantageous triplet for the patient, intervention and robotic tools under consideration. The criteria and their mathematical formulation are detailed in [8], they consist of: reachability, dexterity, patient trauma, surgeon effort and factors related to the operative setup. Note that this approach does not depend on the characteristics of the robot, but only on the endoscopic tools. In fact, the same method is currently being used for classical endoscopic interventions (nephrectomy and prostatectomy). Variations to the above configuration can be accommodated either by bypassing the optimization process or adapting it. For instance a fourth port is sometimes used to pull or stabilize an anatomical entity, in which case a segregation in the target vector can be used to assign different role to different tools, while the overall mechanism remains unchanged. 

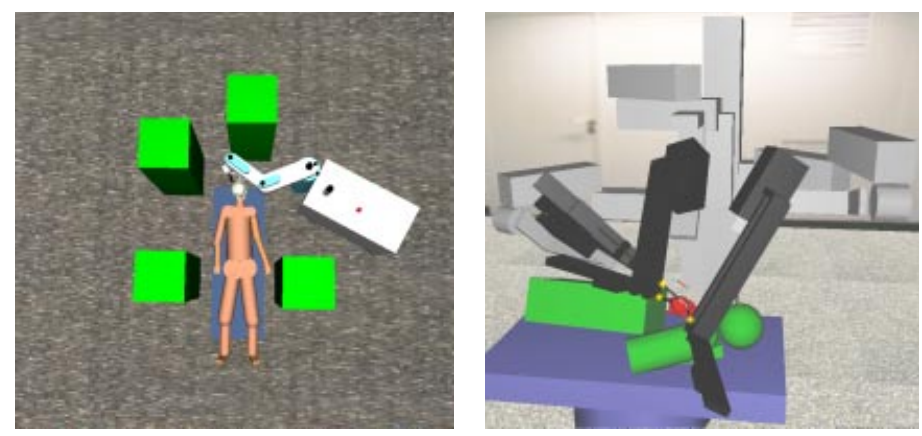

Fig. 4. Pose planning for optimal robot positioning of the SCALPP [11] robot (left) and the Da Vinci ${ }^{\mathrm{TM}}$ system (right).

Experimental trials on plastic models have already shown encouraging results [9], and animal trials are currently being conducted to validate and improve the criteria in use.

\section{Robot Positioning}

Positioning a surgical robot in a typically cluttered operating room for optimal operation is a challenging task. Surgical manipulators are generally designed to have a flexible structure to adapt to the operating environment, and thus have a high number of degrees of freedom (dofs) to position. In mini-invasive interventions, they are also usually subject to additional spatial constraints which are to fixed points that correspond to the entry ports on the patient's skin. Moreover, the planned results should not only guarantee a collision free operation, but should also target a maximum separation form the surrounding obstacles, since this is a critical application, and since the positioning is done in an error prone environment.

A systematic method for the positioning of the passive dofs of a robotic system has been developed and is detailed in [10. Passive dofs are those not under direct tele-operation or autonomous control, they include the position of the base with respect to the operating environment and the patient, and may also include additional setup joints, as illustrated in figure 4 (b) for the latter and (a) for the former.

\section{Verification}

As the CIS systems become more complex and integrated, the risks that accompany such an evolution are inevitably increasing. In particular, both the logical behavior and the processing capabilities of the systems tend to become difficult 
to analyze. The main goal behind this verification step that precedes the intervention is to look for problematic states in which the system could be trapped. A preemptive approach based on a synchronous finite state machine representation is used. The major difficulty resides in finding a unified representation for robotic and surgical actions that will be used to rigorously describe the state of the patient-surgeon-environment. Preliminary analysis is being undertaken to adapt previous work in calssical robotics [12] and assess the needs for modifying the existing approach to better fit medical robotics. Figure 7 shows an example of the logical flow of the registration step (see section 6) implemented using the EsTEREL language 13. Moreover, the same state machines can also be used as a safe supervision mechanism between the executing parts of the system (those in direct contact with the patient) and the rest of the interactive inputs (see section 7). Algorithmic aspects of critical parts of the system (e.g. collision detection) are formally proved using a logical assistant system [14], thus guaranteeing correct behavior both in planning and while execution.

\section{Simulation}

Simulation serves the two subtly different goals of training and rehearsal of surgical procedures. Simulation for training concentrates on teaching surgeons the different interventional strategies, as well as getting them accustomed to the surgical tools they will be using. Standard pathological cases and physiological complications can be included in instructive scenarios. On the other hand, simulation for rehearsal is a patient dependent validation and preparation of planned goals in settings that come as close as possible to those of the operating theater. This kind of simulation gives the surgeon the possibility to try out different strategies for his intervention, different tools, different positions of the patient, etc .. Naturally, patient dependent teaching is also possible. For the purpose of RMIS, rehearsal simulation is more interesting; however, it is more difficult to achieve. Indeed, the non-uniformity of the data requires a large amount of pre-processing to give results as good as those found in a pre-modeled and pre-calculated simulation system. Therefore, realism is often compromised for efficiency and accuracy.

Standard 3d input devices (space mice, PHANTOM ${ }^{\mathrm{TM}}$ arms or the original input console of the system) are used to control the robots, whereas external and endoscopic views are provided through an OpenGL ${ }^{\circledR}$ rendering interface with possible stereoscopic output. Interference detection is possible between the robot and any modeled obstacle, which can include other parts of the robot (e.g. multiple arms) or modeled parts of the patient. Interaction between the tools and the segmented anatomical entities range from simple destruction (e.g. figure 51) to elastic deformation using techniques from [15]. Active constraints can also be added using scalar distance fields around the regions of interest. These operations require an increased level of data pre-processing. 

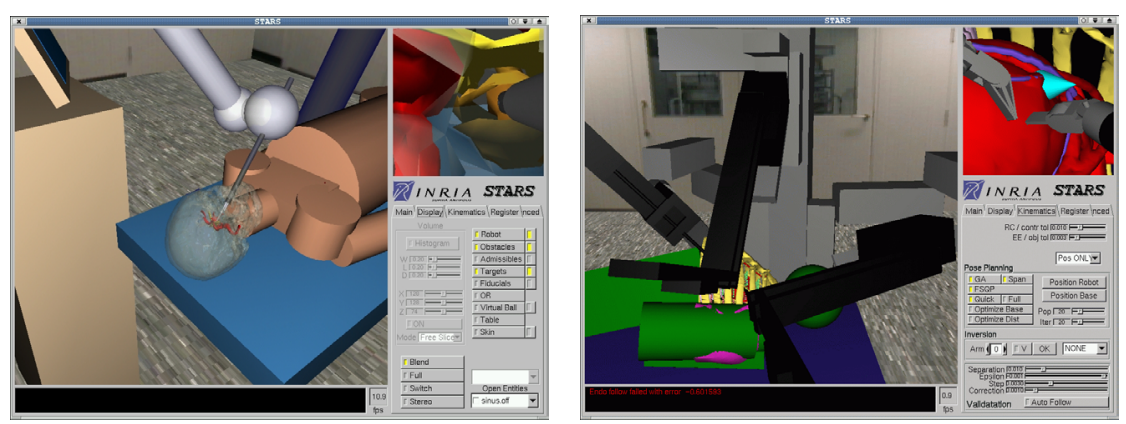

Fig. 5. (left) A simulation of removing a tumor in the pituitary gland using prototyped endoscopic instruments, (right) the Da Vinci ${ }^{\mathrm{TM}}$ robot in a coronary artery bypass graft simulation.

\section{Transfer}

Precision is one of the most important advantages robots bring into the operating room. However, what we are looking for is patient benefit; i.e., both precision and accuracy 2 in the accomplishment of either the preplanned tasks or the operated movements of the robot. This consolidation is made possible through registration between preoperative and intraoperative models, as well as between the robot, the patient and the environment. Moreover, during the intervention the accuracy should constantly be monitored through proper tracking.

A fiducial based least squares registration is used to register the robot to the patient. Precisions of $3 \mathrm{~mm}$ have been observed while registering the Da Vinci ${ }^{\mathrm{TM}}$ robot to a pre-scanned plastic phantom. Animal experiments are currently being conducted to assess the amount error introduced between preoperative and intraoperative conditions, on which tracking method will be used to decrease the effect.

\section{$7 \quad$ Execution and Monitoring}

On-line interference evaluation and augmented reality are major safety and efficiency additions to MIS robotic systems. A minimum separation between critical parts of the robotic system is constantly monitored during the intervention, enabling proper preventive actions to be taken (e.g. alarm system). This simple safety test is being evolved to a logical supervision system using the techniques introduced in section 4. At the same time the surgeon is guided through intraoperative clues that help in endoscopic navigation and offer him an overlay of pertinent preoperative information.

2 Precise not accurate: 

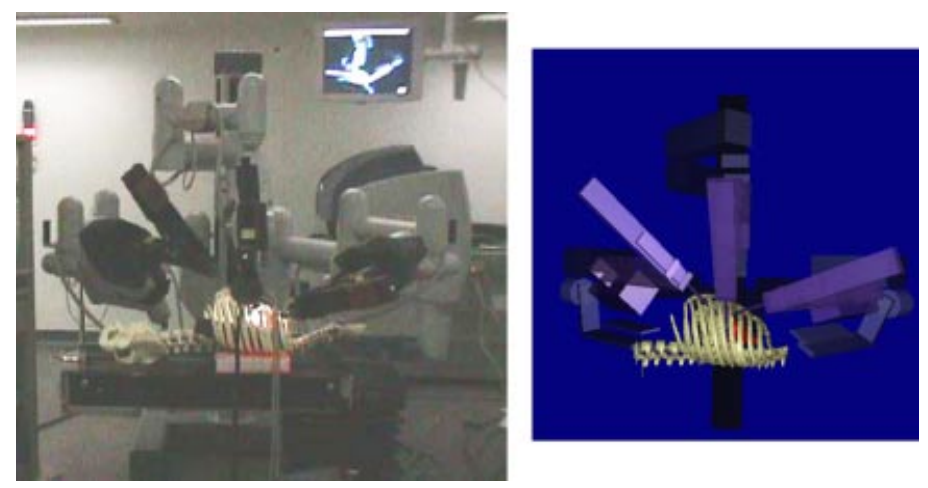

Fig. 6. Transferring planned results to the operating theater after proper registration steps.
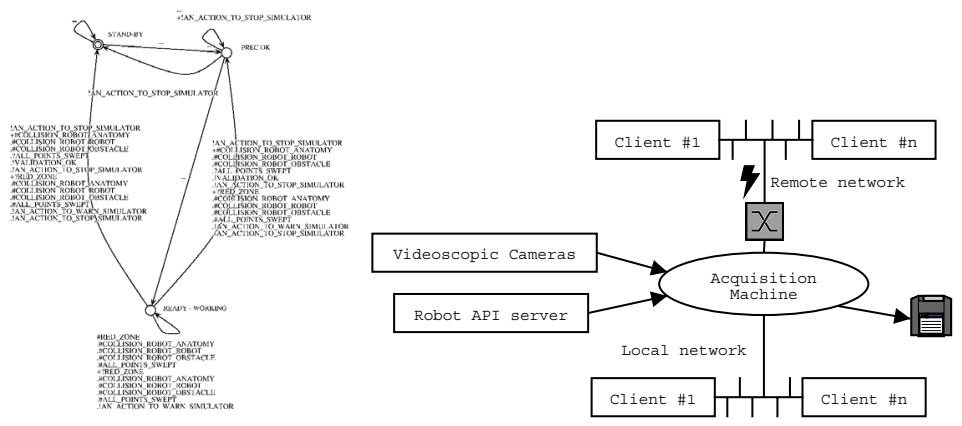

Fig. 7. (left) Part of a finite state machine for verification and monitoring, (right) Intraoperative data acquisition setup for local or remote processing.

Both the monitoring and execution help can be carried out locally or from a remote location provided appropriate throughput and delay times are respected. Figure 7 shows the setup used for acquiring both robotic and image data during the intervention. The acquisition system serves as a transparency layer between low level API drivers and higher level data access commands from the clients. This setup has been implemented for the Da Vinci ${ }^{\mathrm{TM}}$ system and used on the hospital LAN and between the hospital in Paris and the research center in Sophia-Antipolis $(1000 \mathrm{~km}$ distance with $1 \mathrm{Gbits} / \mathrm{s}$ throughput and a nominal delay of $10 \mathrm{~ms}$ ).

\section{Analysis}

The settings and history of the intervention are conserved by recording the articular values of the robot, the endoscopic images and all the registration information between the patient, the robot and the rest of the modeled entities in the 
operating theater. The intervention can then be "replayed", shared, criticized, etc .. On the long run, the major advantage of such an analysis will appear. Namely, the planning results can be correlated to the outcome of the interventions and revisited in order to improve the optimization. The same argument also applies of the rest of the execution steps, where critical chains can be isolated and enhanced.

\section{Conclusions and Perspectives}

An overview of the architecture for a computer integrated minimally invasive robotic surgery has been presented. The underlying implementation (Simulation and Training Architecture for Robotic Surgery: STARS) is characterized by its versatility and modularity in what concerns both robotics and medical image processing. STARS is an integrated CIS system that is used for all the steps described in this paper, ranging from radiological image manipulation to intraoperative registration, going through planning and simulation performed with the surgeon.

Experimental validations on artificial data and phantoms have already shown positive results, and animal tests are currently being conducted to better asses the feasibility of the approach. Future work will evolve around experimental validation which will most surely lead to changes and improvements in all of the constituents of the proposed architecture.

\section{References}

1. Guthart, G., Salisbury Jr., J.K.: The intuitive telesurgery system: Overview and application. In: Proceedings of the 2000 IEEE International Conference on Robotics and Automation. (2000)

2. Tombropoulos, R.Z., Adler, J.R., Latombe, J.C.: Carabeamer: A treatment planner for a robotic radiosurgical system with general kinematics. Medical Image Analysis 3 (1999)

3. Chiu, A.M., Dey, D., Drangova, M., Boyd, W.D., Peters, T.M.: 3-d image guidance for minimally invasive robotic coronary artery bypass. Heart Surgery Forum (2000)

4. Tabaie, H., Reinbolt, J., Graper, P., Kelly, T., Connor, M.: Endoscopic coronary artery bypass graft (ECABG) procedure with robotic assistance. The Heart Surgery Forum 2 (1999)

5. Kühnapfel, U., Çakmak, H., Maaß, H.: 3D modeling for endoscopic surgery. In: Proc. IEEE Symposium on Simulation, Delft University, Delft, NL (1999)

6. Grimson, E., Leventon, M., Ettinger, G., Chabrerie, A., Ozlen, F., Nakajima, S., Atsumi, H., Kikinis, R., Black, P.: Clinical experience with a high precision imageguided neurosurgery system. Lecture Notes in Computer Science 1496 (1998)

7. Taylor, R.: Computer-integrated surgery: Coupling information to action in 21st century medicine. In: IEEE Int. Conf. on Robotics and Automation. (2001)

8. Adhami, L., Coste-Manière, È., Boissonnat, J.D.: Planning and simulation of robotically assisted minimal invasive surgery. In: Proc. Medical Image Computing and Computer Assisted Intervention (MICCAI'00). Volume 1935. (2000) 
9. Coste-Manière, È., Adhami, L., Severac-Bastide, R., Lobontiu, Adrian Salisbury, K., Boissonnat, J.D., Swarup, N., Guthart, G., Mousseaux, É., Carpentier, A.: Optimized port placement for the totally endoscopic coronary artery bypass grafting using the da Vinci robotic system. In: Lecture Notes in Control and Information Sciences, Experimental Robotics VII. Volume 271. (2001)

10. Adhami, L., Coste-Manière, È.: Positioning tele-operated surgical robots for collision-free optimal operation. In: IEEE Int. Conf. on Robotics and Automation. (2002)

11. Duchemin, G., Dombre, E., Pierrot, F., Poignet, P.: Skin harvesting robotization with force control. In: Proc. 10th International Conference on Advanced Robotics. (2001)

12. Borrelly, J.J., Coste-Manière, E., Espiau, B., Kapellos, K., Pissard-Gibollet, R., Simon, D., Turro, N.: The Orccad architecture. International Journal of Robotics Research 17 (1998)

13. Berry, G.: The Esterel v5 language primer. CMA, Écoles des Mines de Paris and INRIA. (2000)

14. Huet, G., Kahn, G., Paulin-Mohring, C.: The Coq Proof Assistant : A Tutorial : Version 7.2. (2002)

15. Cotin, S., Delingette, H., Ayache, N.: Real-time elastic deformations of soft tissues for surgery simulation. IEEE Transactions On Visualization and Computer Graphics 5 (1999) 